\title{
Multidimensional Schrödinger Equation and Spectral Properties of Heavy-Quarkonium Mesons at Finite Temperature
}

\author{
M. Abu-Shady \\ Department of Applied Mathematics, Faculty of Science, Menoufia University, Shbien El-Kom, Egypt \\ Correspondence should be addressed to M. Abu-Shady; dr.abushady@gmail.com
}

Received 17 June 2016; Revised 21 September 2016; Accepted 27 September 2016

Academic Editor: Emmanuel Lorin

Copyright (C) 2016 M. Abu-Shady. This is an open access article distributed under the Creative Commons Attribution License, which permits unrestricted use, distribution, and reproduction in any medium, provided the original work is properly cited.

\begin{abstract}
The $N$-radial Schrödinger equation is analytically solved at finite temperature. The analytic exact iteration method (AEIM) is employed to obtain the energy eigenvalues and wave functions for all states $n$ and $l$. The application of present results to the calculation of charmonium and bottomonium masses at finite temperature is also presented. The behavior of the charmonium and bottomonium masses is in qualitative agreement with other theoretical methods. We conclude that the solution of the Schrödinger equation plays an important role at finite temperature that the analysis of the quarkonium states gives a key input to quark-gluon plasma diagnostics.
\end{abstract}

\section{Introduction}

The Schrödinger equation (SE) plays an important role in describing many phenomena related to the vibrations of diatomic molecules and the oscillations of atoms, high energy physics, and quantum chemistry. Thus, the solutions of the SE are important for describing the phenomena in the abovementioned fields. In [1-9], the authors obtained different solutions of the SE. It is well known that the exact solutions of the SE are found in few cases due to the complexity of the centrifugal potential. Therefore, there are different methods suggested such as those in [10-12], in which the authors solved the SE by using the Nikiforov-Uvarov method. Other authors used asymptotic iteration methods such as in [13-16]. In addition, it is well known that the potential interaction energy of the SE is necessary to obtain the explicit solutions of the SE and the energy eigenvalues, such as the Cornell potential as in $[16,17]$, the extended Cornell potential [18], and the Hulthén plus ringed-shaped potential as in [19].

At finite temperature, in [3], the authors employed the modified internal potential as a function of temperature to study the quark-gluon plasma using Mayer's expansion and a phenomenological thermodynamic model. In [20], the finite temperature SE is solved by using the Funke-Hecke theorem and is applied to electron and proton systems. In [21], the authors obtained the generalized form of the SE at finite temperature based on the first law of thermodynamics. In [22], the authors numerically solved the SE at finite temperature by employing temperature-dependent effective potential given by a linear combination of color-singlet and internal energies. Matsui and Satz [23] have studied the formation of a hot quark-gluon plasma by studying the effect of the temperature on the $J / \Psi$ radius calculated in the charmonium models. Wong [24] has studied the binding energies and wave functions of heavy quarkonia in quark-gluon plasma by using the color-singlet free energy and total internal energy for a static quark and antiquark in quenched QCD. Thus, the study shows that the model with the new $Q-\bar{Q}$ potential gives dissociation temperatures that agree with the spectral function analyses. Additionally, Wong [25] has investigated the $Q-\bar{Q}$ potential by using the thermodynamic quantities to give spontaneous dissociation temperatures for quarkonium and has also found the quark drip lines which separate the region of bound color-singlet $Q \bar{Q}$ states from the unbound region. Reik and Rapp [26] have studied the evaluation of quarkonium bound-state properties and heavy-quark diffusion. They have applied the thermodynamic $T$-matrix approach for elastic two-body interactions to obtain the spectral functions of heavy-quark systems in the quark-gluon plasma, in which the spectral functions are used to calculate Euclidean correlators, which are discussed in light of lattice QCD results. 
On the other hand, studies of lattice QCD at finite temperature with improved actions have provided consistent estimates of $T_{c}$, playing an essential role in investigating the heavy quark. The lattice QCD with two flavors of nonperturbatively improved Wilson fermions at finite temperature is studied to describe the heavy-quark potential [27]. The Debye screening between two opposite color charges is shown in the QCD static potential computed at finite temperature with lattice QCD $[28,29]$. Therefore, the heavy-quark bound states may no longer exist well above the deconfinement critical temperature $T_{c}$ on the order of $200-300 \mathrm{MeV}$ [30].

The aim of the present work is to obtain the solutions of the Schrödinger equation at finite temperature. So far, no attempt has been made to solve the $N$-radial SE using the AEIM when finite temperature is included. The application is studied on the analysis of the quarkonium states which play an important role in the quark-gluon plasma diagnostics in the heavy-ion collision experiments.

The paper is organized as follows: The $N$-radial SE is solved by using the AEIM in Section 2. The results are discussed in Section 3. The summary and conclusion are presented in Section 4.

\section{The Schrödinger Equation at Finite Temperature}

The SE for two particles interacting via a symmetric potential in $N$-dimensional space takes the form as in [31]:

$$
\begin{aligned}
& {\left[\frac{d^{2}}{d r^{2}}+\frac{(N-1)}{r} \frac{d}{d r}-\frac{L(L+N-2)}{r^{2}}+2 \mu(E-V(r))\right]} \\
& . \Psi(r)=0,
\end{aligned}
$$

where $L, N$, and $\mu$ are the angular momentum quantum number, the dimensionality number, and the reduced mass for the quarkonium particle, respectively. Setting the wave function $\Psi(r)=R(r) / r^{(N-1) / 2}$, the following radial Schrödinger equation is obtained:

$$
\begin{aligned}
& {\left[\frac{d^{2}}{d r^{2}}\right.} \\
& \left.\quad+2 \mu\left(E-V(r, T)-\frac{(L+(N-2) / 2)^{2}-1 / 4}{2 \mu r^{2}}\right)\right] \\
& \quad \cdot R(r)=0 .
\end{aligned}
$$

The $V(r, T)$ can be taken as the internal energy potential [3]:

$$
V(r, T)=F_{1}(r, T)-T \frac{\partial F_{1}(r, T)}{\partial T},
$$

where

$$
F_{1}(r, T)=\left(c r-\frac{4}{3} \frac{\alpha_{s}(T)}{r}\right) e^{-m_{D}(T) r},
$$

where $c$ is a free parameter and $\alpha_{s}(T)$ is the running coupling constant which is given by

$$
\alpha_{s}(T)=\frac{2 \pi}{\left(11-(2 / 3) n_{f}\right) \ln \left(T / \beta T_{c}\right)},
$$

where $n_{f}$ is the number of quark flavors, $T_{c}$ is the critical temperature, and $\beta=0.104 \pm 0.009$. The Debye screening mass $m_{D}(T)$ is given by

$$
m_{D}(T)=4 \pi \eta c_{\sigma} \alpha_{s}(T) T
$$

where $\eta$ and $c_{\sigma}$ are parameters of the model. By substituting (3) into (2), we obtain

$$
\begin{aligned}
& {\left[\frac{d^{2}}{d r^{2}}+d_{1}+d_{2} e^{-m_{D}(T) r}+\frac{d_{3}}{r} e^{-m_{D}(T) r}+d_{4} r e^{-m_{D}(T) r}\right.} \\
& \left.\quad+d_{5} r^{2} e^{-m_{D}(T) r}-\frac{(L+(N-2) / 2)^{2}-1 / 4}{r^{2}}\right] R(r) \\
& \quad=0,
\end{aligned}
$$

where

$$
\begin{aligned}
& d_{1}=2 \mu E, \\
& d_{2}=\frac{8 \mu T}{3} \alpha_{s}(T) \frac{d}{d T} m_{D}(T), \\
& d_{3}=\frac{8 \mu \alpha_{s}(T)}{3}+\frac{16 \mu \pi\left(11-(2 / 3) n_{f}\right)}{3\left[\left(11-(2 / 3) n_{f}\right) \ln \left(T / \beta T_{c}\right)\right]^{2}}, \\
& d_{4}=-2 \mu c, \\
& d_{5}=2 \mu c T \frac{d}{d T} m_{D}(T) .
\end{aligned}
$$

By taking the following form of the wave function as in [1]

$$
R_{n l}(r)=f_{n}(r) e^{g_{l}(r)},
$$

where

$$
\begin{aligned}
& f_{n}(r)= \begin{cases}1 & n=0 \\
\prod_{i=1}^{n}\left(r-\alpha_{i}\right) & n=1,2,3, \ldots,\end{cases} \\
& g_{l}(r)=-\frac{1}{2} \alpha r^{2}-\beta r+\delta \ln r ; \quad \alpha>0, \beta>0,
\end{aligned}
$$

$f_{n}(r)$ represents the Laguerre polynomials. By taking the second derivative of (11), we obtain

$$
\begin{aligned}
& R_{n l}^{\prime \prime}(r)=\left(g_{l}^{\prime \prime}(r)+g_{l}^{\prime 2}(r)+\frac{f_{n}^{\prime \prime}(r)+2 g_{l}^{\prime}(r) f_{n}^{\prime}(r)}{f_{n}(r)}\right) \\
& \cdot R_{n l}(r) .
\end{aligned}
$$

Comparing (14) and (7),

$$
\begin{aligned}
d_{1}+ & d_{2} e^{-m_{D}(T) r}+\frac{d_{3}}{r} e^{-m_{D}(T) r}+d_{4} r e^{-m_{D}(T) r} \\
& +d_{5} r^{2} e^{-m_{D}(T) r}-\frac{(L+(N-2) / 2)^{2}-1 / 4}{r^{2}} \\
= & -\left(g_{l}^{\prime \prime}(r)+g_{l}^{\prime 2}(r)+\frac{f_{n}^{\prime \prime}(r)+2 g_{l}^{\prime}(r) f_{n}^{\prime}(r)}{f_{n}(r)}\right) .
\end{aligned}
$$


2.1. Calculation of Energy $E_{0 l}$. Calculating the energy $E_{0 l}$ at $n=0$, where $f_{0}(r)=1$, using (13), and using the expansion $e^{-m_{D}(T) r}=\sum_{i=0}^{n}\left(\left(-m_{D}(T) r\right)^{i} / i !\right)$, we obtain

$$
\begin{aligned}
\left(d_{1}+\right. & \left.d_{2}-m_{D}(T) d_{3}\right)+\frac{1}{r} d_{3} \\
& +\left(d_{4}+d_{3} m_{D}^{2}-d_{2} m_{D}\right) r \\
& +\left(d_{5}+\frac{d_{2}}{2} m_{D}^{2}-d_{4} m_{D}\right) r^{2} \\
& +\left(\frac{1}{2} d_{4} m_{D}^{2}-d_{5} m_{D}\right) r^{3}+\frac{1}{2} d_{5} m_{D}^{2} r^{4} \\
& -\frac{(L+(N-2) / 2)^{2}-1 / 4}{r^{2}} \\
= & -\left(\alpha^{2} r^{2}+2 \alpha \beta r-(\alpha+2 \delta)\right)+\beta^{2}-\frac{2 \beta \delta}{r} \\
& +\frac{\delta(\delta-1)}{r^{2}} .
\end{aligned}
$$

By comparing the coefficients of the powers of $r$ on both sides, we obtain

$$
\begin{aligned}
-d_{5}-\frac{d_{2}}{2} m_{D}^{2}+d_{4} m_{D} & =\alpha^{2} \\
-d_{4}-d_{3} m_{D}^{2}+d_{2} m_{D} & =2 \alpha \beta \\
d_{1}+d_{2}-m_{D} d_{3} & =(\alpha+2 \delta)-\beta^{2}, \\
d_{3} & =2 \beta \delta \\
d_{4} & =0 \\
d_{5} & =0 \\
1 & =\delta(\delta-1) .
\end{aligned}
$$

From (19) and using the formula $d_{1}=2 \mu E$ in (8), we obtain

$$
E_{0 l}=\frac{1}{2 \mu}\left[\alpha(1+2(\delta+0))-\beta^{2}-d_{2}+m_{D}(T) d_{3}\right]
$$

where the parameters $\alpha, \beta$, and $\delta$ are obtained from (17), (18), and (21) as follows:

$$
\begin{aligned}
& \alpha=\sqrt{\left|d_{4} m_{D}-d_{5}-\frac{d_{2}}{2} m_{D}^{2}\right|}, \\
& \beta=\frac{\left|d_{2} m_{D}-d_{4}-d_{3} m_{D}^{2}\right|}{2 \sqrt{\left|d_{4} m_{D}-d_{5}-\left(d_{2} / 2\right) m_{D}^{2}\right|}}, \\
& \delta=\frac{1 \pm \sqrt{1+4\left((L+(N-2) / 2)^{2}-1 / 4\right)}}{2} .
\end{aligned}
$$

2.2. Calculation of Wave Function at $n=0$. We can write (11) at $n=0$ :

$$
R_{0 l}(r)=N_{0 l} r^{\delta} e^{-(1 / 2) \alpha r^{2}-\beta r},
$$

where $N_{0 l}$ is the normalization constant that can be determined by $\int_{0}^{\infty}\left|R_{0 l}(r)\right|^{2} d r=1$. By using [32], we obtain

$$
N_{0 l}=\frac{(2 \alpha)^{(2 \delta+1) / 4} e^{-\beta^{2} / 4 \alpha}}{\sqrt{\Gamma(2 \delta+1) D_{-(2 \delta+1)}(\beta \sqrt{2 / \alpha})}},
$$

where $D_{\nu}(z)$ are the parabolic cylinder functions (see [32] and references therein). The parameters $\alpha, \beta$, and $\delta$ are calculated in (23), (24), and (25). To satisfy the boundary conditions at $R_{0 l}(r=0)=0$, the parameter $\delta$ should have a positive value. Hence, we choose the positive sign in (25). In addition, to satisfy the boundary condition at infinity $R_{0 l}(r=\infty)=$ 0 , the parameters $\alpha$ and $\beta$ should have a positive value. Therefore, the absolute values are taken in (23) and (24).

2.3. Calculation of Energy $E_{1 l}$. To calculate the energy eigenvalue $E_{1 l}$, the index $n$ is taken as $n=1$. Thus, (11) is written as

$$
f_{1}(r)=\left(r-\alpha_{1}\right) \text {. }
$$

Therefore, we can write (15) as

$$
\begin{aligned}
& \left(d_{1}+d_{2}-m_{D}(T) d_{3}\right)+\frac{1}{r} d_{3}+\left(d_{4}+d_{3} m_{D}^{2}-d_{2} m_{D}\right) \\
& \cdot r+\left(d_{5}+\frac{d_{2}}{2} m_{D}^{2}-d_{4} m_{D}\right) r^{2}+\left(\frac{1}{2} d_{4} m_{D}^{2}\right. \\
& \left.-d_{5} m_{D}\right) r^{3}+\frac{1}{2} d_{5} m_{D}^{2} r^{4} \\
& -\frac{(L+(N-2) / 2)^{2}-1 / 4}{r^{2}}=-\left(\alpha^{2} r^{2}+2 \alpha \beta r\right. \\
& -\alpha(1+2(\delta+1))+\beta^{2}-\frac{2\left[\beta(\delta+1)+\alpha \alpha_{1}\right]}{r} \\
& \left.+\frac{\delta(\delta-1)}{r^{2}}\right) .
\end{aligned}
$$

By comparing the coefficients of the powers $r$ on both sides, we obtain

$$
\begin{aligned}
-d_{5}-\frac{d_{2}}{2} m_{D}^{2}+d_{4} m_{D} & =\alpha^{2}, \\
-d_{4}-d_{3} m_{D}^{2}+d_{2} m_{D} & =2 \alpha \beta, \\
d_{1}+d_{2}-m_{D}(T) d_{3} & =\alpha(1+2(\delta+1))-\beta^{2}, \\
d_{3} & =2\left[\beta(\delta+1)+\alpha \alpha_{1}\right], \\
d_{4} & =0 \\
d_{5} & =0 \\
-\frac{1}{4} & =\delta(\delta-1) .
\end{aligned}
$$


From (31) and using the formula $d_{1}=2 \mu E$ in (8), we obtain $E_{1 l}$ as the following form:

$$
E_{1 l}=\frac{1}{2 \mu}\left[\alpha(1+2(\delta+1))-\beta^{2}-d_{2}+m_{D}(T) d_{3}\right] .
$$

2.4. Calculation of Wave Function at $n=1$. We can write the wave function at $n=1$ :

$$
R_{1 l}(r)=N_{1 l}\left(r-\alpha_{1}\right) r^{\delta} e^{-(1 / 2) \alpha r^{2}-\beta r}
$$

where the parameter $\alpha_{1}$ is obtained from (32):

$$
\alpha_{1}=\frac{d_{3}-2 \beta(\delta+1)}{2 \alpha}
$$

where the parameters $\alpha, \beta$, and $\delta$ are those given in (23), (24), and (25). $N_{1 l}$ is the normalization constant, which can be determined by $\int_{0}^{\infty}\left|R_{1 l}(r)\right|^{2} d r=1$. Thus, we obtain

$$
N_{1 l}=\frac{1}{\sqrt{I_{1}-2 \alpha_{1} I_{2}+\alpha_{1}^{2} I_{3}}},
$$

where

$$
\begin{aligned}
& I_{1}=\frac{\Gamma(2 \delta+3) D_{-(2 \delta+3)}(\beta \sqrt{2 / \alpha})}{(2 \alpha)^{\delta+3 / 2} e^{-\beta^{2} / 2 \alpha}} \\
& I_{2}=\frac{\Gamma(2 \delta+2) D_{-(2 \delta+2)}(\beta \sqrt{2 / \alpha})}{(2 \alpha)^{\delta+1} e^{-\beta^{2} / 2 \alpha}} \\
& I_{3}=\frac{\Gamma(2 \delta+1) D_{-(2 \delta+1)}(\beta \sqrt{2 / \alpha})}{(2 \alpha)^{\delta+1 / 2} e^{-\beta^{2} / 2 \alpha}} .
\end{aligned}
$$

2.5. Calculation of Energy $E_{2 l}$. Following the analytic iteration method for the second node $n=2$,

$$
f_{2}(r)=\left(r-\alpha_{1}\right)\left(r-\alpha_{2}\right)
$$

we can write (15), and using (13), we obtain

$$
\begin{aligned}
& \left(d_{1}+d_{2}-m_{D}(T) d_{3}\right)+\frac{1}{r} d_{3}+\left(d_{4}+d_{3} m_{D}^{2}-d_{2} m_{D}\right) \\
& \cdot r+\left(d_{5}+\frac{d_{2}}{2} m_{D}^{2}-d_{4} m_{D}\right) r^{2}+\left(\frac{1}{2} d_{4} m_{D}^{2}\right. \\
& \left.-d_{5} m_{D}\right) r^{3}+\frac{1}{2} d_{5} m_{D}^{2} r^{4}-\frac{(L+(N-2) / 2)^{2}-1 / 4}{r^{2}} \\
& =-\left(\alpha^{2} r^{2}+2 \alpha \beta r-\alpha(1+2(\delta+2))-\beta^{2}\right. \\
& \left.-\frac{2\left[\beta(\delta+2)+\alpha \sum_{i=1}^{2} \alpha_{i}\right]}{r}+\frac{\delta(\delta-1)}{r^{2}}\right) .
\end{aligned}
$$

The relations between the potential parameters and the coefficients $\alpha, \beta, \delta, \alpha_{1}$, and $\alpha_{2}$ are as follows:

$$
\begin{aligned}
-d_{5}-\frac{d_{2}}{2} m_{D}^{2}+d_{4} m_{D} & =\alpha^{2}, \\
-d_{4}-d_{3} m_{D}^{2}+d_{2} m_{D} & =2 \alpha \beta, \\
d_{1}+d_{2}-m_{D}(T) d_{3} & =\alpha(1+2(\delta+2))+\beta^{2}, \\
d_{3} & =2\left[\beta(\delta+2)+\alpha \sum_{i=1}^{2} \alpha_{i}\right], \\
d_{4} & =0, \\
d_{5} & =0, \\
\left(L+\frac{(N-2)}{2}\right)^{2}-\frac{1}{4} & =\delta(\delta-1) .
\end{aligned}
$$

From (44) and using the formula $d_{1}=2 \mu E$ in (8), we obtain $E_{2 l}$ :

$$
E_{2 l}=\frac{1}{2 \mu}\left[\alpha(1+2(\delta+2))+\beta^{2}-d_{2}+m_{D}(T) d_{3}\right] .
$$

\subsection{Calculation of Wave Function at $n=2$}

$$
R_{2 l}(r)=N_{2 l}\left(r-\alpha_{1}\right)\left(r-\alpha_{2}\right) r^{\delta} e^{-(1 / 2) \alpha r^{2}-\beta r},
$$

where $\alpha_{1}$ and $\alpha_{2}$ are obtained from (45) and (37). $N_{2 l}$ is the normalization constant which can be obtained as in (38).

2.7. Exact Energy and Wave Function. The iteration method is repeated. Therefore, we obtain the exact energy at finite temperature as the following form:

$$
E_{n l}=\frac{1}{2 \mu}\left[\alpha(1+2(\delta+n))-\beta^{2}-d_{2}+m_{D}(T) d_{3}\right],
$$

and the wave function is

$$
R_{n l}(r)=N_{n l} \prod_{i=1}^{n}\left(r-\alpha_{i}\right) r^{\delta} e^{-(1 / 2) \alpha r^{2}-\beta r},
$$

where the parameters $\alpha, \beta$, and $\delta$ are defined in (23), (24), and (25). $N_{n l}$ is the normalization constant that can be obtained as in (27) and (38).

\section{Discussion of Results}

In the section above, the $N$-Schrödinger equation is solved at finite temperature by using the AETM as in [1]. The parameters of the present work are shown in Table 1. In this section, we apply the energy eigenvalue that is given in (49). To calculate quarkonium masses at finite temperature, the formula $M=2 m+E_{n l}$ is used when $N=3$, where $m$ is bare quark mass.

It is important to apply the present results on quarkonium mesons. In [33], the authors have investigated the 
TABLE 1: The parameters of the internal energy.

\begin{tabular}{lc}
\hline Parameter & Value [3] \\
\hline$\beta$ & 0.104 \\
$c_{\sigma}$ & 0.566 \\
$\eta$ & 2.06 \\
$T_{c}$ & $0.25 \mathrm{GeV}$ \\
$c$ & $0.135 \pm 0.015 \mathrm{GeV}^{2}$ \\
\hline
\end{tabular}

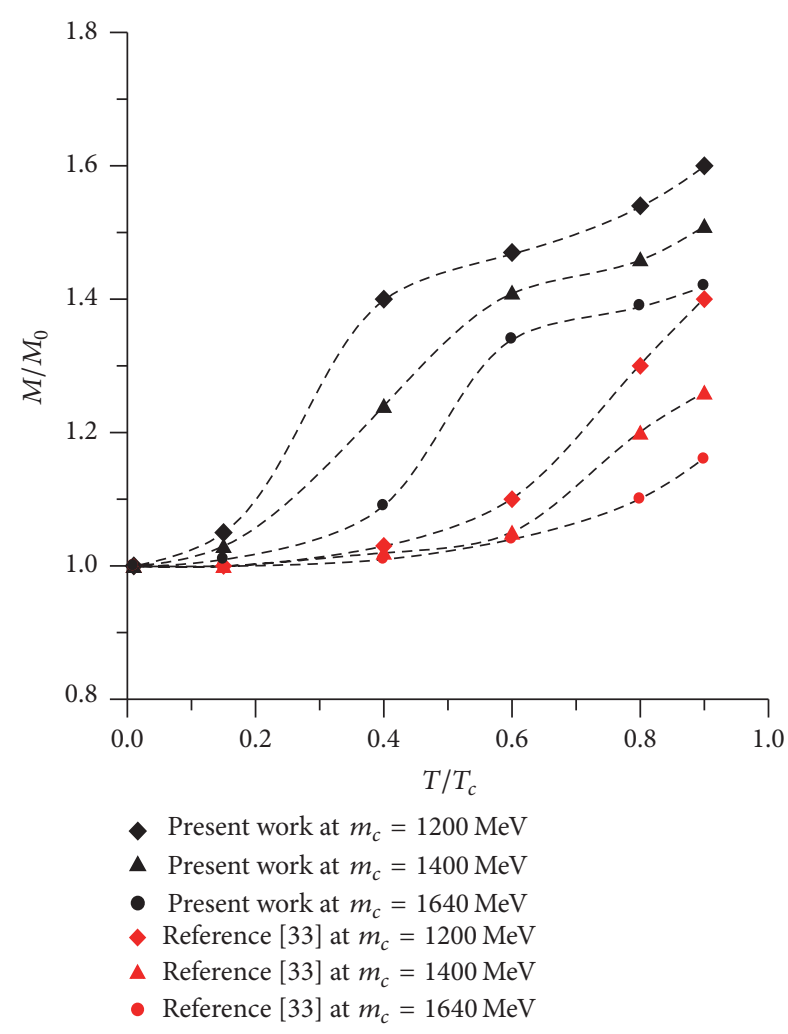

FIGURE 1: Mass in vacuum mass units of charmonium ground state is a function of temperature in critical temperature units for charm quark mass $\left(m_{c}\right)$ equal to 1200,1400 , and $1640 \mathrm{MeV}$.

quarkonium spectrum calculations at finite temperature in the framework of QCD sum-rules. They found that the $1 S$ state of quarkonium mass changes little up to $T / T_{c}=0.2\left(T_{c}\right.$ is critical temperature equal to $250 \mathrm{MeV})$; then, the behavior of charmonium mass increases with increasing temperature up to $T / T_{c}=1$. In addition, the curves shift to lower values by increasing charm mass $\left(m_{c}\right)$ as in Figure 1 .

Also in Figure 1, we find that the $1 S$ state of charmonium mass little changes up to $T / T_{c}=0.2$ and then the charmonium mass increases with increasing temperature. The curves shift to lower values by increasing $m_{c}$ in the present study. Additionally, we note that the present results of charmonium mass are shifted to upper values in comparison with [33], since the parameters of each method are changed. So, we note that qualitative agreement between the present results and [33]. The study of Debye mass has much interest in studying quarkonium properties. Unfortunately, there is still quite small diversity of results in this quantity. In Figure 2,
TABLE 2: The screening mass in the lattice QCD with $N_{f}=3.0$ and the present work.

\begin{tabular}{lcc}
\hline Ratio temperature $\left(T / T_{c}\right)$ & 1.5 & 2 \\
Lattice QCD with $N_{f}=3.0$ & 1.40 & 2.34 \\
The present work $N_{f}=3.0$ & 1.34 & 1.76 \\
\hline
\end{tabular}

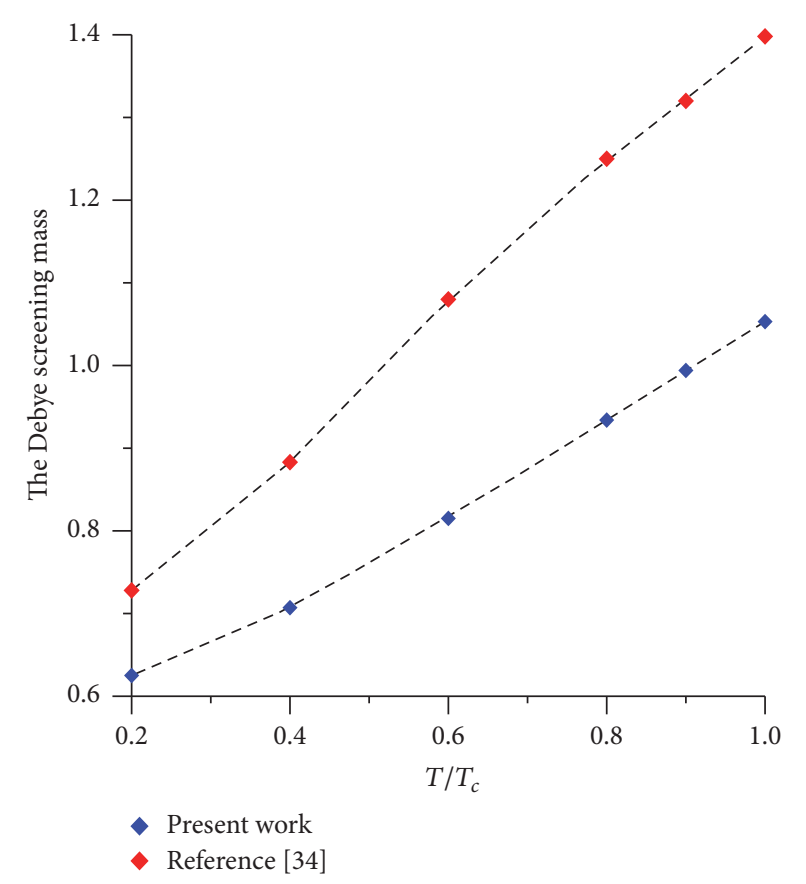

FIgURE 2: The Debye screening mass is a function of ratio temperature at $m_{c}=1640 \mathrm{MeV}$.

the comparison between the Debye screening mass in the present work and its value in [34] is presented. We find that qualitative agreement is noted in comparison with [34]. Additionally, lattice QCD with $N_{f}=3$ is studied in this quantity [35]. Table 2 shows the comparison between the present results of screening mass and the lattice QCD. We note that the screening mass is in good agreement with lattice QCD at $T / T_{c}=1.5$ and the screening mass is shifted to a higher value at $T / T_{c}=2.0$.

\section{Summary and Conclusion}

In the present work, the solutions of $N$-radial Schrödinger equation are obtained for all states $n$ and $l$, where the energy eigenvalue and wave function are obtained at finite temperature. The analytic exact iteration method (AEIM) in [1] is used as the technique for solving the SE. The novelty in this work is that we obtain the analytic solution of the $N$-radial $\mathrm{SE}$ at finite temperature by using the AEIM. In addition, the energy eigenvalues and corresponding wave functions are calculated in the $\mathrm{N}$-dimensional space, in which one obtains the energy eigenvalue and wave function in the 3-dimensional space which are used in the most of the other works.

We apply the theoretical calculations on the quarkonium masses at finite temperature. We find that the behavior of 
quarkonium masses is in qualitative agreement in comparison with the QCD sum rule and lattice QCD, which are tools for measuring quarkonium masses at quark-gluon plasma. Therefore, the present approach successfully describes the quarkonium states for the given potential, which are a key input to quark-gluon plasma. We hope to extend this work to include electromagnetic forces in future work.

\section{Competing Interests}

The author declares no competing interests.

\section{References}

[1] S. Özçelik and M. Şimşek, "Exact solutions of the radial Schrödinger equation for inverse-power potentials," Physics Letters A, vol. 152, no. 3-4, pp. 145-150, 1991.

[2] M. Znojil, "Singular anharmonicities and the analytic continued fractions. II. The potentials $V(r)=a r^{2}+b r^{-4}+c r^{-6}$," Journal of Mathematical Physics, vol. 31, p. 108, 1990.

[3] N. M. El Naggar, L. I. Abou Salem, A. G. Shalaby, and M. A. Bourham, "The equation of state for non-ideal quark gluon plasma," Physical Science International Journal, vol. 4, no. 7, pp. 912-929, 2014.

[4] S. M. Ikhdair and R. Sever, "An alternative simple solution of the sextic anharmonic oscillator and perturbed Coulomb problems," International Journal of Modern Physics C, vol. 18, no. 10, pp. 1571-1581, 2007.

[5] L. I. Abou-Salem, "Mass spectra and leptonic decay widths of heavy quarkonia by using psi function," Journal of Physics G: Nuclear and Particle Physics, vol. 30, no. 10, pp. 1391-1397, 2004.

[6] S. M. Kuchin and N. V. Maksimenko, "Characteristics of charged pions in the quark model with potential which is the sum of the Coulomb and oscillator potential," Journal of Theoretical and Applied Physics, vol. 7, no. 1, article 47, 2013.

[7] S. M. Ikhdair, B. J. Falaye, and M. Hamzavi, "Nonrelativistic molecular models under external magnetic and AB flux fields," Annals of Physics, vol. 353, pp. 282-298, 2015.

[8] B. J. Falaye, S. M. Ikhdair, and M. Hamzavi, "Spectroscopic study of some diatomic molecules via the proper quantization rule," Journal of Mathematical Chemistry, vol. 53, no. 6, pp. 1325-1350, 2015.

[9] B. J. Falaye, K. J. Oyewumi, F. Sadikoglu, M. Hamzavi, and S. M. Ikhdair, "Analysis of quantum-mechanical states of the ringshaped Mie-type diatomic molecular model via the Fisher's information," Journal of Theoretical and Computational Chemistry, vol. 14, no. 5, Article ID 1550036, 2015.

[10] H. Eğrifes, D. Demirhan, and F. Büyükkılıç, "Exact solutions of the Schrödinger equation for the deformed hyperbolic potential well and the deformed four-parameter exponential type potential," Physics Letters A, vol. 275, no. 4, pp. 229-237, 2000.

[11] S. M. Ikhdair, "On the bound-state solutions of the ManningRosen potential including an improved approximation to the orbital centrifugal term," Physica Scripta, vol. 83, no. 1, Article ID 015010, 2011.

[12] M. Hamzavi, K. Thylwe, and A. Rajabi, "Approximate bound states solution of the Hellmann potential," Communications in Theoretical Physics, vol. 60, no. 1, pp. 1-8, 2013.
[13] B. Falaye, "Arbitrary $\ell$-state solutions of the hyperbolical potential by the asymptotic iteration method," Few-Body Systems, vol. 53, no. 3-4, pp. 557-562, 2013.

[14] B. J. Falaye, K. J. Oyewumi, T. T. Ibrahim, M. A. Punyasena, and C. A. Onate, "Bound state solutions of the Manning-Rosen potential," Canadian Journal of Physics, vol. 91, no. 1, pp. 98-104, 2013.

[15] B. J. Falaye, S. M. Ikhdair, and M. Hamzavi, "Formula method for bound state problems," Few-Body Systems, vol. 56, no. 1, pp. 63-78, 2015.

[16] S. M. Kuchin and N. V. Maksimenko, "Theoretical estimations of the spin-averaged mass spectra of heavy quarkonia and Bc mesons," Universal Journal of Physics and Application, vol. 1, no. 3, pp. 295-298, 2013.

[17] H. S. Chung, J. Lee, and D. Kang, "Cornell potential parameters for S-wave heavy quarkonia," Journal of the Korean Physical Society, vol. 52, pp. 1151-1154, 2008.

[18] N. V. Masksimenko, S. M. Kuchin, and J. Russ. Phy, 2014.

[19] H. Hassanabadi, S. Zarrinkamar, and H. Rahimov, "Approximate solution of D-dimensional Klein-Gordon equation with Hulthén-type potential via SUSYQM," Communications in Theoretical Physics, vol. 56, no. 3, p. 423, 2011.

[20] G. P. Malik, R. K. Jha, and V. S. Varma, "Finite-temperature Schrödinger equation: solution in coordinate space," The Astrophysical Journal, vol. 503, no. 1, pp. 446-449, 1998.

[21] X. Y. Wu, B. Zhang, X. Liu, Y. Wu, Q. Wang, and Y. Wang, "Finite temperature Schrödinger equation," International Journal of Theoretical Physics, vol. 50, no. 8, pp. 2546-2551, 2011.

[22] W. M. Alberico, A. Beraudo, A. D. Pace, and A. Molinari, "Quarkonia in the deconfined phase: effective potentials and lattice correlators," Physical Review D, vol. 75, no. 7, Article ID 074009, 2007.

[23] T. Matsui and H. Satz, " $J / \psi$ suppression by quark-gluon plasma formation," Physics Letters B, vol. 178, no. 4, pp. 416-422, 1986.

[24] C.-Y. Wong, "Heavy quarkonia in quark-gluon plasma," Physical Review C, vol. 72, no. 3, Article ID 034906, 2005.

[25] C.-Y. Wong, "Quarkonia and quark drip lines in a quark-gluon plasma," Physical Review C, vol. 76, no. 1, Article ID 014902, 2007.

[26] F. Reik and R. Rapp, "Quarkonia and heavy-quark relaxation times in the quark-gluon plasma," Physical Review C, vol. 82, Article ID 035201, 2010.

[27] V. Bornyakov, M. Chernodub, Y. Koma et al., "Heavy quark potential in lattice QCD at finite temperature," https://arxiv.org/ abs/hep-lat/0301002.

[28] F. Karsch and H. W. Wyld, "Screening of the QCD heavy quark potential at finite temperature," Physics Letters B, vol. 213, no. 4, pp. 505-510, 1988.

[29] F. Karsch, "News from lattice QCD on heavy quark potentials and spectral functions of heavy quark states," Journal of Physics G: Nuclear and Particle Physics, vol. 30, no. 8, pp. S887-S894, 2004.

[30] F. Karsch, "Lattice results on QCD thermodynamics," Nuclear Physics A, vol. 698, no. 1-4, pp. 199-208, 2002.

[31] R. Kumar and F. Chand, "Asymptotic study to the N-dimensional radial schrödinger equation for the quark-antiquark system," Communications in Theoretical Physics, vol. 59, no. 5, pp. 528-532, 2013.

[32] S. M. Ikhdair and M. Hamzavi, "Spectral properties of quantum dots influenced by a confining potential model," Physica B: Condensed Matter, vol. 407, no. 24, pp. 4797-4803, 2012. 
[33] F. S. Navarra and C. A. A. Nunes, "Temperature dependence of heavy meson masses," Physics Letters B, vol. 356, no. 4, pp. 439444, 1995.

[34] S. Gao, B. Liu, and W. Q. Chao, "Color screening and dissociation of charmonium at finite temperature and finite density," Physics Letters B, vol. 378, no. 1-4, pp. 23-28, 1995.

[35] F. Karsch, M. T. Mehr, and H. Satz, "Color screening and deconfinement for bound states of heavy quarks," Zeitschrift für Physik C Particles and Fields, vol. 37, no. 4, pp. 617-622, 1988. 


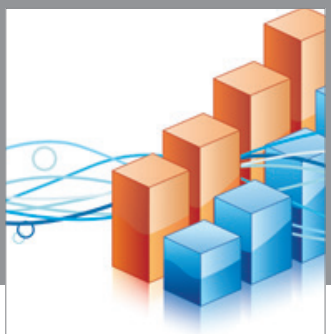

Advances in

Operations Research

vatem alat4

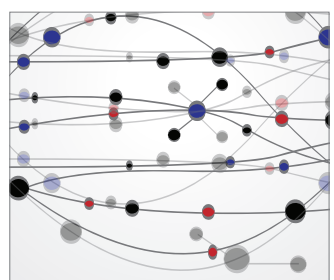

\section{The Scientific} World Journal
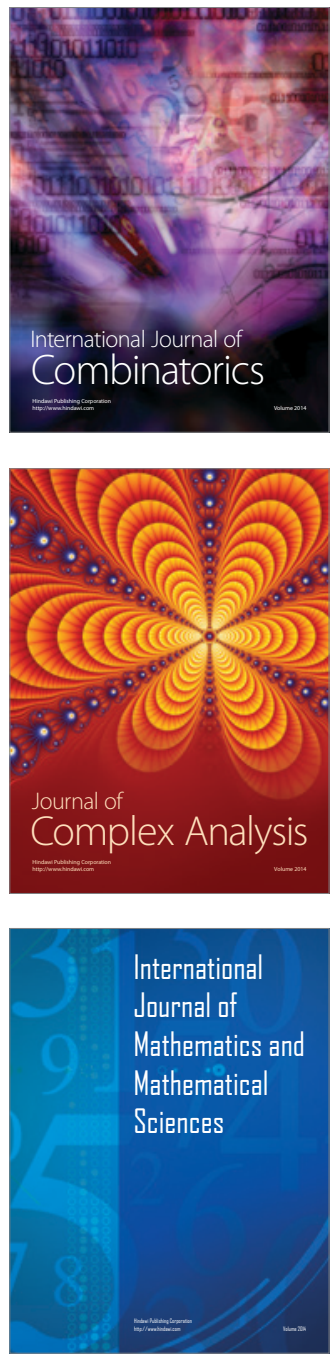
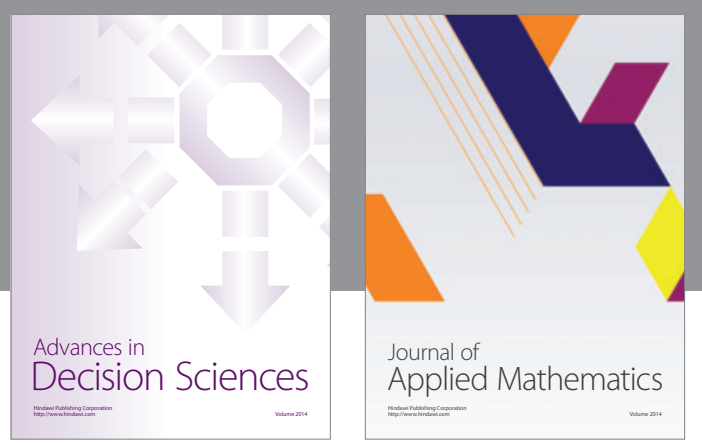

Algebra

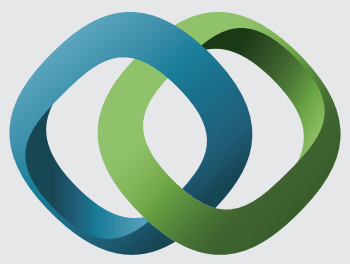

\section{Hindawi}

Submit your manuscripts at

http://www.hindawi.com
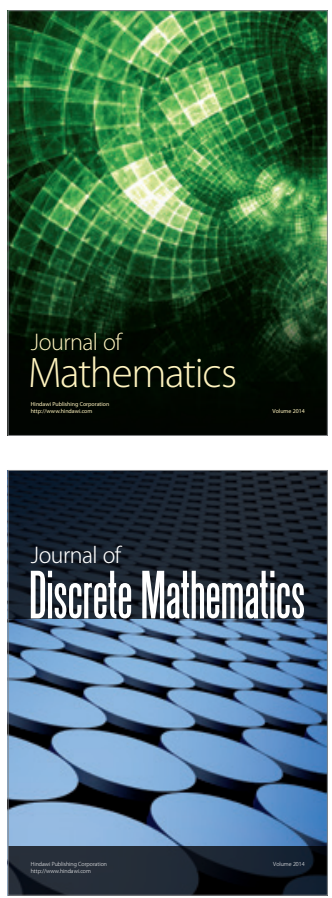

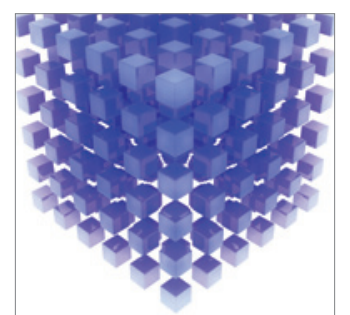

Mathematical Problems in Engineering
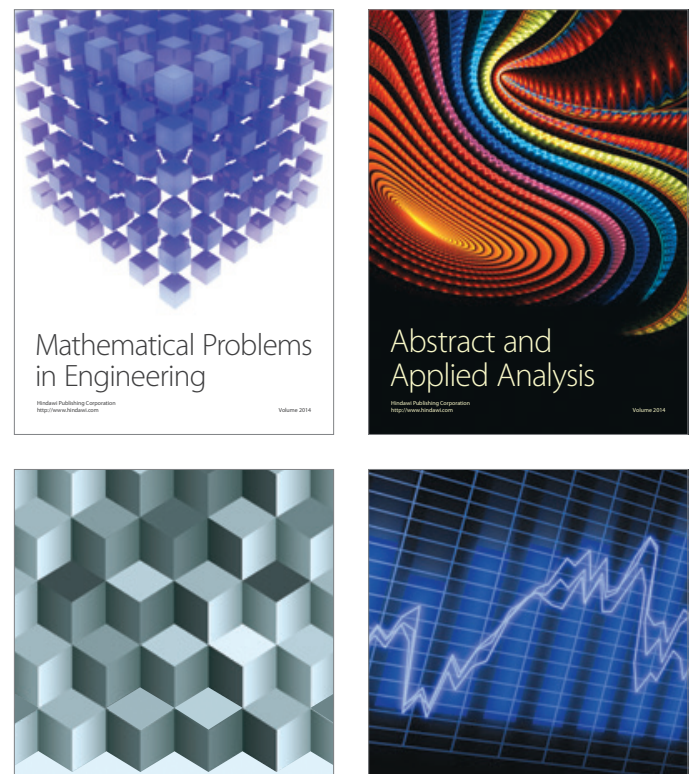

Journal of

Function Spaces



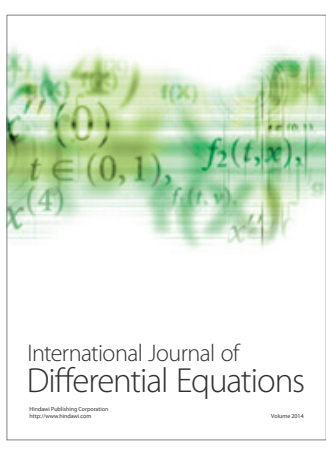
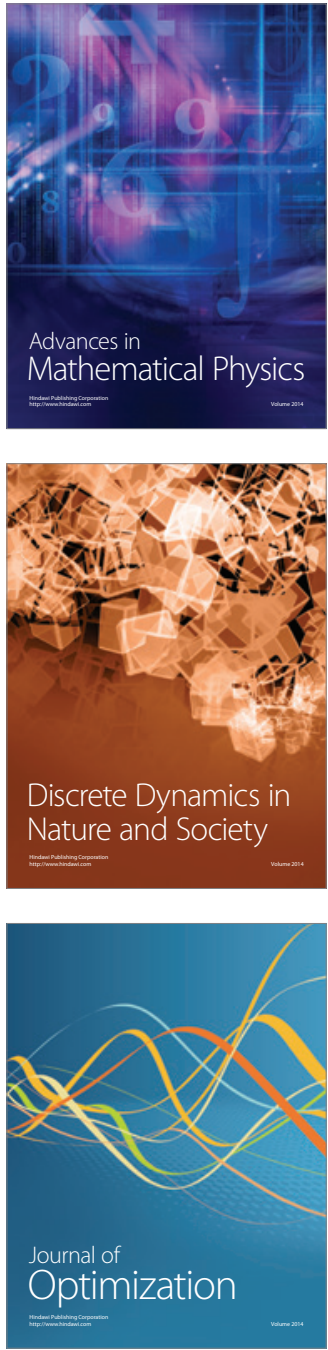